\title{
Adapting Physical Carrier Sensing to Maximize Spatial Reuse in 802.11 Mesh Networks
}

Jing Zhu*, Xingang Guo, L. Lily Yang, W. Steven Conner, Sumit Roy†, and Mousumi M. Hazra

\{jing.a.zhu, xingang.guo, lily.l.yang, w.steven.conner,

mousumi.m.hazra\}@intel . com

Communications Technology Lab

Intel Corporation

2111 NE 25 th Ave., JF3-206

Hillsboro, OR 97124, U.S.A.

${ }^{*}$ Contact author (for proofing), currently with Dept. of Electrical Engineering, U. Washington. Email: zhuj@ee.washington.edu; Address: Department of Electrical Engineering, University of Washington Box 352500 Seattle, WA 98195-2500 USA; Phone: 206-618-2846. This work was completed while he was an intern at Intel Corporation.

${ }^{\dagger}$ Currently with Dept. of Electrical Engineering, U. Washington; roy@ee.washington.edu. This work was completed while he was on leave at Intel Corporation. 


\begin{abstract}
Spatial reuse in a mesh network can allow multiple communications to proceed simultaneously, hence proportionally improve the overall network throughput. To maximize spatial reuse, the MAC protocol must enable simultaneous transmitters to maintain the minimal separation distance that is sufficient to avoid interference. This paper demonstrates that physical carrier sensing enhanced with a tunable sensing threshold is effective at avoiding interference in 802.11 mesh networks without requiring the use of virtual carrier sensing. We present an analytical model for deriving the optimal sensing threshold given network topology, reception power, and data rate. A distributed adaptive scheme is also presented to dynamically adjust the physical carrier sensing threshold based on periodic estimation of channel conditions in the network. Simulation results are shown for large-scale $802.11 \mathrm{~b}$ and $802.11 \mathrm{a}$ networks to validate both the analytical model and the adaptation scheme. It is demonstrated that the enhanced physical carrier sensing mechanism effectively improves network throughput by maximizing the potential of spatial reuse. With dynamically tuned physical carrier sensing, the end to end throughput approaches $90 \%$ of the predicted theoretical upper-bound assuming a perfect MAC protocol, for a regular chain topology of 90 nodes.
\end{abstract}

Key words: 802.11, MAC, Physical Carrier Sensing, and Adaptive Algorithm.

\title{
1 Introduction
}

Over the past few years we have witnessed the rapid proliferation of wireless LANs in various network environments (home, office and public hotspots). The need for higher data rates and improved coverage has led to at least two potential solutions for large-scale WLANs - a) multi-cell networks where each cell is serviced by its own access point (AP), and b) mesh networks where nodes work in ad-hoc mode and use multi-hop routing to relay each other's traffic. In both cases, the overall network throughput is proportional to the number of simultaneous communications via co-channel spatial reuse that can be conducted in spatially separated locations with acceptable mutual interference.

A multi-cell WLAN is similar to a traditional cellular network whereby spatial reuse is achieved through careful site planning and engineered channel assignment for each cell. In an ad-hoc network, however, no access point or base station infrastructure exists and hence engineered channel assignment 
is often not feasible. Furthermore, because of the random topology of ad-hoc networks, detecting and avoiding interference is also more complicated. In [4], spatial reuse was demonstrated to depend on various characteristics of the network, including the type of radio, network topology, channel quality requirements and signal propagation environment. For each network configuration, there exists a minimum separation distance such that when simultaneous transmitters are separated by that distance, the maximum number of simultaneous transmissions can be accommodated, leading maximum network throughput. However, achieving maximum spatial reuse would require an ideal MAC protocol that schedules communication to maintain the optimal transmitter separation distance in a fully distributed manner while minimizing interference.

Nodes using the IEEE 802.11 MAC protocol [1] use carrier sensing to determine if the shared medium is available before transmitting to avoid packet collision. Two types of carrier sensing are supported by the 802.11 MAC: mandatory physical carrier sensing (PCS) that monitors the RF energy level in the channel and optional virtual carrier sensing (VCS) that uses the Request-to-Send/Clear-to-Send (RTS/CTS) handshake to effectively reserve the channel prior to data transmission. Virtual carrier sensing was designed to avoid the well-known hidden terminal problem [9], where it is assumed that physical carrier sensing at a transmitter is not sufficient to avoid interference at a receiver. Interestingly, a substantial portion of existing literature on .11 interference management is based on RTS/CTS. This has it's limitations; the overhead due to the additional RTS/CTS handshake is not justified when the data payload size is small. More significantly, it has been shown that virtual carrier sensing has fundamental limitations in avoiding interference from hidden terminals in mesh networks [7] [10] [11] [12], i.e. there are many scenarios where it is conservative and fails to suppress packet collisions as intended.

Trying to resolve this by making RTS/CTS more aggressive exacerbates the exposed terminal problem, whereby feasible parallel transmissions that do not interfere with the reference one are suppressed.

The above prompts a re-evaluation of the role and effectiveness of PCS in interference management; in this paper we demonstrate that when properly tuned, PCS is effective at avoiding interference in a multi-hop wireless mesh network, without the use of VCS. PCS allows a station to assess the channel condition before transmitting to make sure that no interference can occur. A station samples the energy level in the medium and starts a packet 
transmission only if the reading is below the carrier sensing threshold, indicating that no simultaneous transmissions are taking place that could result in interference to the desired. Using RF pathloss models, the carrier sensing threshold may be translated into an effective minimum allowed distance between simultaneous transmitters. As noted, this optimal distance depends on various network properties; thus the carrier sensing threshold should be tuned to match network conditions. However, many of today's 802.11 MAC implementations use a static threshold, or do not allow the threshold to be independently tunable [14]. As a result, physical carrier sensing often leads transmitters to be either too conservative or too aggressive due to improper setting of the threshold and is the likely reason why use of PCS has not attracted much attention.

In this work, we make the case for a tunable carrier sensing threshold; some simple analysis is used to derive the appropriate carrier sensing threshold for given network topology. Furthermore, we propose an estimation-based adaptive PCS scheme to automatically tune the threshold to a near-optimal value. We present OPNET simulation results for two regular network topologies (chain and grid) to validate the theoretical PCS threshold. Our results further show that by tuning the PCS threshold, the overall network throughput can be improved significantly compared to that of legacy 802.11 MAC without any VCS. Furthermore, the throughput can approach approximately $90 \%$ of the theoretical upper-bound predicted by spatial reuse models in a large chain. Simulation results also demonstrate the effectiveness of the estimation-based adaptive PCS scheme in networks with dynamic topology and heterogeneous links.

It is worth noting that throughput of 802.11 networks may be enhanced by attending to many different aspects of the $802.11 \mathrm{MAC}$ protocol; this is the subject of extensive work, such as [7] [8] [12] to cite a few. Hence maximizing network performance must be a careful combination of approaches addressing multiple aspects (e.g. collision resolution, fairness, etc.) of the network behavior. The focus of this paper is purely on leveraging the spatial reuse in mesh networks to enhance the throughput through PCS, which we believe is particulary apropos in a dense in-building ad-hoc MESH network where the nodes are either static or have only intermittent mobility.

The rest of this paper is organized as follows: Section 2 presents the SINR communication model that is used in this paper for our interference analysis and the limitations of carrier in 802.11 DCF. Section 3 introduces our analytical model for PCS and shows how a tunable physical sensing threshold can 
dramatically improve the throughput of mesh networks with regular topologies. Section 4 describes our novel estimation-based adaptive physical carrier sensing scheme. Section 5 presents OPNET simulation results demonstrating measurable benefit from tuned physical carrier sensing and the adaptation scheme. Finally the paper is concluded in section 6 .

\section{Managing Interference with Carrier Sensing}

In CSMA/CA protocol, the transmitter relies on carrier sensing to determine if the medium is 'available' for channel access. A station samples the energy level on the channel and a transmission is only started if the energy level is below the PCS threshold.

\subsection{Communication Model}

A path loss model expresses the average signal strength at the receiver as a function of the T-R (transmitter-receiver) separation distance, $d$, i.e.

$$
P_{r x}(d)=\bar{P}_{r x}-\gamma \log _{10}(d / \bar{d}) \quad(\text { all powers in } \mathrm{dB})
$$

where $\gamma$ is the path loss exponent ${ }^{1}$ at distance $d$ from the transmitter and $\bar{P}_{r x}$ is the reference signal strength as measured at the distance $\bar{d}$ (usually 1 meter).

The aggregate energy detected by a receiver consists of signal (from intended transmitter), interference (from unwanted transmitter(s)) and background noise. A receiver can receive a packet with acceptable error rate only if two conditions are satisfied: i) the received desired signal is greater than a threshold (denoted by $P_{R}$, i.e. receiver sensitivity) in the noise only case, and ii) the Signal-Noise-Interference Ratio (SNIR) is above a threshold (denoted by $\left.S_{0}\right)$ in the presence of multiple access interference.

$$
\left\{\begin{array}{l}
P_{r x}(d) \geq P_{R} \\
\frac{P_{r x}(d)}{P_{N}+\sum_{i} P_{r x}\left(d_{i}\right)} \geq S_{0}
\end{array},\right.
$$

where $P_{N}$ is the strength of the background noise, and $P_{r x}\left(d_{i}\right)$ denotes the signal strength from interference source $i$ at distance $d_{i}$. 802.11 networks

\footnotetext{
${ }^{1} \gamma=2$ for a free-space LOS (Line-Of-Sight) model and $\gamma=4$ for a ground reflection (two-ray) model. The issue of how to choose $\gamma$ for different indoor environments at $5.0 \mathrm{GHz}$ has been studied experimentally in [5].
} 
support multiple data rates, and a higher data rate typically requires a higher threshold $S_{0}$.

It is clear from Eq.2 that successful reception at any T-R separation depends on the received SINR; hence any definition of transmission range for a given communication depends on the local network (interference) environment. However as a starting point, we define the transmission range based only on the receiver sensitivity $P_{R}$; i.e. the transmission range is the maximum T-R separation for successful reception in additive noise only.

Fig. $1^{2}$ shows a segment in a typical mesh network with a reference transmission from a TX node to a RX node and four other neighboring nodes (A, $\mathrm{B}, \mathrm{C}$, and $\mathrm{E}$ ), where the same transmission power is used by every node. We define the following:

$D$ : T-R separation distance, such that $P_{D}=P_{r x}(D)$.

$R$ : Transmission range, given by

$$
R=\bar{d}\left(\frac{\bar{P}_{r x}}{\max \left(P_{R}, S_{0} P_{N}\right)}\right)^{\frac{1}{\gamma}}=\bar{d}\left(\frac{\bar{P}_{r x}}{P_{R}}\right)^{\frac{1}{\gamma}},
$$

$I$ : Interference range: a single transmitter within that range of the receiver will disrupt reception of desired transmisssion, given by

$$
I=D\left(\frac{1}{\frac{1}{S_{0}}-\left(\frac{D}{d}\right) \gamma \frac{P_{N}}{P_{r x}}}\right)^{1 / \gamma} .
$$

With negligible background noise, Eq.4 turns to

$$
I \approx S_{0}^{1 / \gamma} D
$$

$X$ : Physical carrier sensing range - a node will be able to detect an existing transmitter within that range via physical carrier sensing, given by

$$
X=\bar{d}\left(\frac{\bar{P}_{r x}}{P_{C}}\right)^{\frac{1}{\gamma}},
$$

where $P_{C}$ denotes the physical carrier sensing (PCS) threshold.

Table 1 briefly summarizes the common symbols used throughout this paper to describe carrier sensing.

\footnotetext{
${ }^{2}$ The exact relation between the physical carrier sensing range $X$ and the interference range $I$ is determined by Eq.4 and 6. Here, we just show an example with $X>I$.
} 


\begin{tabular}{|l|l|}
\hline$P_{R}$ & Reception Power Threshold \\
$P_{C}$ & Physical Carrier Sensing Threshold \\
$P_{D}$ & Reception Power \\
$P_{N}$ & Background Noise Power \\
$P_{I}$ & Interference Power \\
$S_{0}$ & SNIR Threshold \\
$\gamma$ & Path Loss Exponent \\
$X$ & Physical Carrier Sensing Range \\
$R$ & Transmission Range \\
$I$ & Interference Range \\
$D$ & Transmission Distance \\
$p_{c s} t$ & $P_{C} / P_{D}$ \\
$k$ & Spatial reuse factor \\
$W$ & Link capacity \\
\hline
\end{tabular}

Tab. 1: Common symbols for describing physical carrier sensing.

\subsection{Limitations of Carrier Sensing in 802.11 MAC Protocols}

In today's 802.11 networks, the physical carrier sensing scheme is typically configured with a fixed threshold, which is often set very low such that even a remote transmission would cause the reference station to withhold its transmission. As a result, very little spatial reuse is allowed leading to poor aggregate network throughput. Moreover, the fixed threshold cannot be dynamically tuned according to different environments. As wireless networks are deployed at higher densities in multi-hop mesh topologies, the need to exploit spatial reuse increases; accordingly, adapting the carrier sensing threshold, wherever possible, is key to improving network throughput.

It is also worth noting that in addition to PCS schemes, virtual carrier sensing (VCS) schemes [1] are also used in wireless networks. With VCS, a station maintains its NAV (Network Allocation Vector) that indicates the period(s) during which the channel is reserved by other stations; hence it knows when NOT to transmit. When contending for the medium, a station broadcasts its intended transmission period in the RTS frame (which is also echoed in the CTS). Each station that receives these broadcasts updates its NAV. Hence, VCS requires participating stations to be able to decode the RTS/CTS broadcast frames from any other stations in the network with 
which they may potentially interfere. Unfortunately, this requirement cannot be guaranteed in most dense wireless networks [10]. Fig. 1 demonstrates the limitations of the VCS scheme for preventing interference as was shown in [10]. The VCS scheme can effectively prevent nodes A and B from initiating an interfering transmission, as they are in the transmission range of TX and $\mathrm{RX}$, respectively; however node $\mathrm{C}$ is too far away from both TX and RX to reliably decode the RTS or CTS packets, yet it is still a potential hidden node that could interfere with the packet reception at RX.

\section{Enhancing Physical Carrier Sensing}

\subsection{Tuning Physical Carrier Sensing (PCS) to Avoid Interference}

Physical carrier sensing allows a station to assess the channel conditions before transmitting to avoid interference that will lead to packet collisions. A station samples the net energy at it's air interface and starts a packet transmission only if the sensed energy is below a threshold $P_{C}$, called the PCS threshold. The determinant whether a packet can be successfully received by a receiver is a threshold value of the signal to noise plus interference ratio (SINR) at the receiver. Thus, the goal of PCS via threshold tuning is to prevent simultaneous transmissions that will lead to packet collisions, while permitting simultaneous transmissions that will not violate receiver SNIR requirements so as to maximize spatial reuse.

Fig. 2 illustrates a simple example of how the choice of PCS threshold can impact wireless network performance. If the threshold is too high, the CSMA is more conservative than necessary. As shown in Fig. 2 (a), while node $\mathrm{C}$ is transmitting, both nodes $\mathrm{A}$ and $\mathrm{B}$ will backoff, even though node $\mathrm{A}$ may be able to simultaneously transmit without causing excessive interference at C's receiver to disrupt successful communication. However if in Fig. 2 (b), the threshold is sufficiently high so as to allow both nodes A and B to transmit simultaneously with $\mathrm{C}$, excessive interference will be generated resulting in packet collisions. If the PCS threshold is appropriately configured, as shown in Fig. 2 (c), nodes $\mathrm{A}$ and $\mathrm{C}$ will be permitted to successfully transmit simultaneously while node $\mathrm{B}$ will be forced to back off to prevent packet collisions. When the PCS threshold is optimized, maximal spatial reuse can be achieved without permitting packet collisions. 
When properly tuned, PCS is more robust than the VCS, because it does not require control packets to be received and correctly decoded. It is also more flexible, since the PCS sensing range can be easily adjusted by tuning the PCS threshold. In Fig.1, all potentially interfering nodes, including node $\mathrm{C}$, can be eliminated by enlarging the PCS sensing range to cover the entire potential interference area, i.e.

$$
X \geq D+I
$$

Combining Eq.7 with Eq.5, we obtain

$$
X \geq D\left(1+S_{0}^{1 / \gamma}\right)
$$

that leads to

$$
P_{c s_{-} t} \leq \frac{1}{\left(1+S_{0}^{1 / \gamma}\right)^{\gamma}} .
$$

Another well-known problem occurs due to exposed terminals [8]; for example, even though a transmission by node $\mathrm{E}$ will not disrupt RX, because it is within the sensing range of TX, E will defer its transmission. Having too many exposed terminals can potentially reduce the overall network throughput. By tuning the physical carrier sensing threshold, we will demonstrate a good tradeoff between hidden terminals and exposed terminals so as to obtain high aggregate throughput.

\subsection{Estimating Optimal PCS Threshold to Maximize Spatial Reuse}

We now investigate the choice of the optimal PCS threshold that allows for maximum spatial reuse, by assuming homogeneous wireless links and identical interference and noise environments at all nodes ${ }^{3}$. To initiate channel access by a node, the interference and noise sensed by its receiver cannot exceed the tolerable level according to Eq.2,

$$
P_{I}+P_{N} \leq P_{D} / S_{0},
$$

It therefore follows that the PCS threshold should satisfy

$$
P_{C} \leq P_{D} / S_{0}
$$

\footnotetext{
3 This assumption is clearly the primary weakness of the model.
} 
for successful simultaneous transmissions. Since higher the PCS threshold implies more simultaneous transmissions, $P_{D} / S_{0}$ is the optimal PCS threshold for maximal spatial reuse. The corresponding optimal $p_{c s \_t}$ defined as $\beta$ is seen to be independent of path loss exponent $\gamma$, i.e.,

$$
\beta=\frac{1}{S_{0}}
$$

Let $\rho$ denote the ratio of the exposed terminal area to the whole PCS sensing area with $\frac{1}{\left(1+S_{0}^{1 / \gamma}\right)^{\gamma}}$; then using Eq. 9, this is given by

$$
\rho=\frac{\pi X^{2}-\pi I^{2}}{\pi X^{2}} \approx \frac{D^{2}\left(1+S_{0}^{1 / \gamma}\right)^{2}-D^{2} S_{0}^{2 / \gamma}}{D^{2}\left(1+S_{0}^{1 / \gamma}\right)^{2}}=1-\left(\frac{S_{0}^{1 / \gamma}}{1+S_{0}^{1 / \gamma}}\right)^{2} .
$$

When $S_{0}^{1 / \gamma}$ is small, $\rho$ is not negligible; but with $S_{0}^{1 / \gamma}>>1^{4}$, we have $\rho \approx 0$ so that the exposed terminal problem can be ignored, and Eq.9 turns into $p_{c s \_t} \leq \beta$.

\subsection{Analysis Model for Aggregate Throughput Limits}

In [4] the authors investigated spatial reuse from a physical layer perspective. A homogeneous environment was assumed where every transmitter uses the same transmission power and data rate, and communicates to an immediate neighbor at the constant T-R distance $d$. Under such conditions, spatial reuse can be characterized by the distance between neighboring simultaneous transmitters (minimum T-T separation distance) that results in optimal spatial reuse. The authors investigated the optimal spatial reuse for two regular network topologies: the 1-D chain network and the 2-D grid network. Let $k$ denote the T-T distance (also called spatial reuse factor), in number of hops (hop distance being $d$ ), then the lower bounds of $k$ for the two topologies are

$$
\begin{cases}k \geq\left[2\left(1+\frac{1}{\gamma-1}\right) S_{0}\right]^{\frac{1}{\gamma}}, & \text { Chain network } \\ k \geq\left[6\left(1+\frac{1}{\gamma-2}\right) S_{0}\right]^{\frac{1}{\gamma}}, & 2-\mathrm{D} \text { grid }\end{cases}
$$

If we assume a perfect MAC protocol that schedules simultaneous communications only on transmitters that are $k$ hops away from each other, the

\footnotetext{
${ }^{4}$ More so for higher data rates since higher $S_{0}$ values will be required.
} 
network will be able to accommodate the maximum number of simultaneous transmitters, hence reaching its aggregate throughput limit. Hence the lower bounds of $k$ can be used to extrapolate to aggregate throughput limits. For example, in a chain network of $N$ nodes, where a packet will require relay by each of the $N-2$ intermediate nodes in order to be routed end-to-end, at most $N / k$ simultaneous transmitters can be supported in the chain. Let $C_{t h}$ denote the end-to-end throughput, then

$$
C_{t h} \approx \frac{W}{k}
$$

where $W$ denotes the effective MAC layer data rate achieved at each relay, i.e. link capacity.

\section{An Estimation-based Adaptive Physical Carrier Sensing (APCS) Scheme}

In the previous section, a theoretical estimate for optimal $\beta$ or normalized carrier sensing threshold $p_{c s_{-} t}$ was derived for a spatially homogeneous network. It is difficult to repeat the above analysis for a network with heterogeneous links (i.e. different receiver sensitivities and/or transmit power). In this section, we propose an adaptive scheme which allows each individual station to calculate and self-configure a near-optimal PCS threshold based on its estimate of the current local interference condition. As a result, the entire network is able to achieve the near-optimal aggregate throughput. Furthermore, such distributed adaptation is also designed to make stations adopt the same threshold. Hence, each station will have equal time-share of the channel, resulting in fair usage of network bandwidth.

The objective of the estimation-based adaptation is to allow simultaneous spatially separated transmissions while ensuring that SINR at each station remains above the desired threshold $S_{0}$. This is accomplished by local measurement and statistics exchange between radio neighbors.

Each node keeps track of the following state variables for the purpose of estimation and adaptation:

$T_{e}$ : The periodic duration of estimation and adaptation (seconds)

$\bar{S}(i)$ : The estimation of the average SINR in a duration at the $i$ th update 
$\xi(i)$ : Indicator for adaptation at the $i$ th update (2: Increase, 1: No Change, 0: Decrease)

$P_{C}^{(\min )}(i)$ : The estimation of the minimum $P_{C}(i)$ in the network at the $i$ th update.

and uses a key parameter of the APCS algorithm:

$\delta(i)$ : The one-step adjustment unit at the $i$ th update.

Both $\bar{S}(i)$ and $P_{C}(i)$ are updated only once every $T_{e}$ seconds while, $\xi(i)$ and $P_{C}^{(\min )}(i)$ are updated whenever ACK is received with "More Frag" off. The value of $\bar{S}(i)$ in this interval is the estimate of average SNIR in the previous interval. At the beginning of each interval, we set $\xi(i)$ with respect to $\bar{S}(i)$ as follows

$$
\xi(i)= \begin{cases}2, & \bar{S}(i) / S_{0} \geq \delta \\ 1, & 1 / \delta<\bar{S}(i) / S_{0}<\delta \\ 0, & \bar{S}(i) / S_{0} \leq \delta\end{cases}
$$

and $P_{C}^{(\min )}(i)=P_{C}(i)$.

This scheme requires disseminating the locally measured statistics $\xi(i)$ and $P_{C}^{(\min )}(i)$ throughout the network neighborhood. For easy implementation, we piggyback the information into the 16 bits "Duration ID" field of 802.11 ACK frame when it is not used. Fig.3 shows the header format of 802.11 MAC ACK packet. When overhearing an ACK, a node will check its "More Frag" flag and "Duration ID" field regardless of whether the ACK is destined for the node. In the standard 802.11 MAC protocol, the "Duration ID" field in an ACK frame is used for the purpose of virtual carrier sensing when the "More Frag" flag is set to 1 ( meaning there are more fragments of a packet following). If the ACK is for the last fragment of the packet or fragmentation is not used, the "More Frag" is turned off (set to 0) and "duration ID" field is not used. Hence we can piggyback information in the "Duration ID" field for the estimation-based adaptive PCS scheme when "More Frag" is $1^{5}$.

\footnotetext{
${ }^{5}$ While this implementation was convenient for simulation purposes to avoid potential interoperability problems from non-standard use of ACK frames, it may be preferable to define a new frame format for this purpose.
} 
Let $x$ and $y$ be the value of $\xi(i)$ and $P_{C}^{(\min )}(i)$ carried by that ACK packet, the node updates its own $\xi(i)$ and $P_{C}^{(\min )}(i)$ with

$$
\left\{\begin{array}{l}
\xi(i)=\min (x, \xi(i)) \\
P_{C}^{(\text {min })}(i)=\min \left(y, P_{C}^{(\text {min })}(i)\right)
\end{array} .\right.
$$

At the end of the interval, each node update its own $P_{C}(i)$ as follows:

$$
P_{C}(i)=\left\{\begin{array}{ll}
P_{C}^{(\min )}(i) \times \delta, & (\xi=2) \\
P_{C}^{(\min )}(i), & (\xi=1) \\
P_{C}^{(\min )}(i) / \delta, & (\xi=0)
\end{array} .\right.
$$

The initial value of $P_{C}(i=0)$ for all nodes is set to $P_{R} / S_{0}$, where $P_{R}$ is the receiver sensitivity and results in the minimum value of $P_{D}$. Fig. 4 illustrates the feedback control diagram for the scheme.

\section{Simulation Results and Discussions}

All the simulation experiments reported were conducted in the OPNET simulation environment [13]. Accordingly, we have extended OPNET kernel modules to support tunable physical carrier sensing, a configurable propagation environment and multiple $802.11 \mathrm{~b}$ data rates. In all simulations, we configured each node to be always backlogged with packets to send, and each MAC data frame to be 1024 bytes long. Each node transmits at a fixed power of $0 \mathrm{dbm}$. By default, the OPNET simulator configures the physical carrier sensing threshold to be the same as the reception threshold. Furthermore, the ambient noise level was set at $-200 \mathrm{dBm}$.

The primary performance metric studied in this paper is throughput, defined as the total number of bits successfully received in a second. Due to MAC semantics, if the sender does not receive an intended ACK packet, it assumes that the data packet is lost and performs a retransmission. However, it is also possible that a data packet is received correctly, but the ACK packet is lost. This situation also causes a retransmission and can result in multiple copies of the data packet at the receiver; in this case, only the first will be forwarded up to higher layers, and the duplicates discarded. When computing throughput in this paper, we only count non-duplicate data packets that are successfully received, i.e., goodput, which is less than the actual network throughput. However, since ACK packets are much smaller than 


\begin{tabular}{|c|c|c|c|c|}
\hline Data Rate $(\mathrm{Mbps})$ & 1 & 2 & 5.5 & 11 \\
\hline$S_{0}(\mathrm{~dB})$ & 11 & 14 & 18 & 21 \\
$\mathrm{~W}(\mathrm{Mbps})$ & 0.89 & 1.5 & 3.5 & 5.0 \\
\hline
\end{tabular}

Tab. 2: One-hop performance of 802.11b MAC without RTS/CTS

data packets and are typically transmitted using the lowest (most reliable) data rate in 802.11 , the probability of successfully receiving a data packet but losing an ACK packet is very low. Thus, the throughput is approximately equal to goodput in an 802.11 network.

Although both collision and interference impact on SINR, the primary focus of the simulations in this section are on interference avoidance. The collision avoidance method of the 802.11 MAC protocol is binary exponential backoff $(\mathrm{BEB})$ with a contention window $(\mathrm{CW})$ size parameter. In our simulations, the BEB mechanism is disabled and the contention window size is fixed at the maximum value for $802.11 \mathrm{~b}(\mathrm{CW}=1024)$ to minimize the likelihood of collisions due to simultaneous transmission. This configuration allows us to focus on the specific effects of adaptive physical carrier sensing on network performance, which is the primary focus of this paper.

\subsection{Point-to-point baseline performance of $802.11 \mathrm{~b}$ MAC}

To quantitatively validate the effectiveness of physical carrier sensing, we need the following two baseline figures: the SINR thresholds $\left(S_{0}\right)$ required to sustain each available data rate in an 802.11 b network, and the effective MAC throughput at each data rate. In the first simulation, we configured a network of two nodes - one sender and one receiver. The pathloss exponent was configured to be 2 to reflect a free-space environment. With RTS/CTS disabled, we varied the T-R separation distance and measured the effective throughput provided by the MAC layer at the receiver. The same simulation sequence was repeated for all four data rates defined in the $802.11 \mathrm{~b}$ standard.

The results in Fig.5 show the throughput against the SINR at receiver (in place of T-R separation). This fundamental relationship between MAC throughput and receiver SNIR is valid irrespective of pathloss exponent and transmission power. The results compiled in Table 2, will be used to design and analyze further simulations in the rest of the section. 


\subsection{Maximizing Spatial Reuse with the Optimal PCS}

We conducted simulations with two regular topologies, 90-node linear chain and $10 \times 102-\mathrm{D}$ grid, to validate the theoretical optimal PCS threshold $\beta$ derived in Section 3.2.

In the 90 node chain (that approximates an infinite one), the only traffic allowed is between node 1 (source)- node 90 (destination) pair. The receiver sensitivity $\left(P_{R}\right)$ was configured such that the transmission range is 13 meters. Each packet was relayed by the other 88 intermediate nodes before reaching its destination. The same carrier sensing threshold and data rate were used by every node. We measured the end-to-end throughput while varying the sensing threshold and the data rate. The path loss exponent was configured to be 2 to approximate a free-space propagation environment. The results are plotted in Fig. 6 and shows that there exists an optimal sensing threshold value for each data rate. With all other parameters fixed, altering the data rate changes the SINR requirement $\left(S_{0}\right)$ and consequently the optimal sensing threshold. Also notice that the common practice of having the carrier sense threshold equal to the reception threshold is equivalent to having $P_{c s_{-} t}=0 \mathrm{db}$, which corresponds to the right-most point on respective curves. Hence, the throughput improvement achieved by tuning the PCS threshold can be 4 times that of an non-optimized setting at rate $11 \mathrm{Mbps}$.

Table 3 compares the optimal sensing threshold $p_{c s_{-} t}$ obtained from the simulations against the theoretical optimum $\beta$. As the table shows, the two values match very well.

\begin{tabular}{|c|c|c|c|c|}
\hline Data Rate (Mbps) & 1 & 2 & 5.5 & 11 \\
\hline$\beta$ (theoretical) & -11 & -14 & -18 & -21 \\
Simulation & -11 & -15 & -17 & -19 \\
\hline
\end{tabular}

Tab. 3: Optimal carrier sensing thresholds $(\mathrm{dB})$ in a 90 -node chain

Table 4 compares the throughputs obtained from simulations with optimal PCS threshold setting against the prediction from the spatial reuse study described in Sec.III-C. As shown in Table 4, the optimally tuned physical carrier sensing was able to achieve around $90 \%$ of the theoretical prediction.

Next, we turn to a 2 -D $10 \times 10$ grid, which is more representative of typical real world topologies. Each packet has its own destination chosen randomly from the immediate neighbors of the transmitter. In this configuration, the distance between neighboring nodes was 4.5 meters. The reception power 


\begin{tabular}{|c|c|c|c|c|}
\hline Data Rate (Mbps) & 1 & 2 & 5.5 & 11 \\
\hline$W$ (Mbps) & 0.89 & 1.5 & 3.4 & 5.0 \\
$k$ (spatial reuse) & 7.1 & 10 & 15.9 & 22.4 \\
T: Theoretical $(W / k)$ & 0.105 & 0.15 & 0.21 & 0.223 \\
S: Simulation & 0.1 & 0.134 & 0.185 & 0.196 \\
S/T & $95 \%$ & $89 \%$ & $88 \%$ & $88 \%$ \\
\hline
\end{tabular}

Tab. 4: Optimal E2E throughput in a 90-node chain

threshold $\left(P_{R}\right)$ was configured to allow the transmission range of only 4.5 meters such that only immediate neighbors could directly communicate.

We conducted four sets of simulations using $1 \mathrm{Mbps}, 2 \mathrm{Mbps}$, 5.5 Mbps and $11 \mathrm{Mbps}$ as the data rates for each node, respectively. In each set of the simulations, we altered the path loss exponent and PCS threshold. The aggregate throughputs of the grid network are plotted in Fig. 7. It is evident that the optimal PCS threshold does not change with the path loss exponent in a large homogeneous network, and the simulation optimal PCS threshold matches the theoretical $\beta$ very well (see Table 5 ).

\begin{tabular}{|c|c|c|c|c|}
\hline Data Rate (Mbps) & $\gamma=2$ & $\gamma=2.5$ & $\gamma=3$ & $\beta$ (theoretical) \\
\hline 1 & -11 & -11 & -11 & -11 \\
2 & -13 & -13 & -13 & -14 \\
5.5 & -17 & -17 & -17 & -18 \\
11 & -19 & -20 & -20 & -21 \\
\hline
\end{tabular}

Tab. 5: Simulation results of optimal carrier sensing thresholds $(d B)$ in a $10 \times 10802.11 b$ grid

To further compare the effectiveness of PCS vis-a-vis VCS, we enabled the VCS with $p_{c s_{-} t}=0 d B$, and measured the aggregate throughput to that when optimal PCS threshold is used (without any VCS operational) for 1 Mbps links. The results in Fig. 8 show that tunable PCS has a decisive advantage over VCS that carries over to other data rates as well.

We also conducted a similar simulation for 802.11a ${ }^{6}$. Table 6 compares the theoretical optimal $p_{\text {cs_t }}$ (i.e. $\beta$ ) with the optimal value from simulations, showing that the analytical estimate of the optimal carrier sensing threshold $\beta$ is also valid for 802.11a network.

\footnotetext{
${ }^{6}$ We use the same modulation curve for 802.11a simulation as in [16]
} 


\begin{tabular}{|c|c|c|c|c|c|c|c|c|}
\hline Data Rate (Mbps) & 6 & 9 & 12 & 18 & 24 & 36 & 48 & 54 \\
\hline$\beta$ (theoretical) & -7 & -9 & -11 & -13 & -17 & -22 & -27 & -29 \\
Simulation & -7 & -9 & -11 & -13 & -17 & -21 & -27 & -29 \\
\hline
\end{tabular}

Tab. 6: Optimal carrier sensing thresholds $(\mathrm{dB})$ in a $10 \times 10802.11 \mathrm{a}$ grid $(\gamma=3)$

\subsection{Evaluation of Adaptive PCS}

Now that the benefits of tuning PCS have been demonstrated, we investigate the performance of the proposed adaptive PCS scheme. First, we study the effect of adaptation when the network topology changes. For this scenario, a simulation is started with a $10 \times 10$ grid using the configuration described in Section 5.2. Midway through the experiment, a subset of the nodes in the network are disabled such that only the nodes in the $8 \times 8$ grid in the center of the network continue generating traffic. In the following simulations, the APCS algorithm is configured with an adaptation duration $\left(T_{e}\right)$ of one second and a one-step adjustment unit $(\delta)$ of $0.5 \mathrm{~dB}$.

Fig. 9 shows the traces for both aggregate throughput and the adaptively tuned PCS threshold $p_{c s_{-} t}$ in the simulation where the topology is changed from a $10 \times 10$ grid to an $8 \times 8$ grid after 50 seconds. It is clear that the adaptive PCS scheme adjusted the PCS threshold as the network conditions changed. Compared to the baseline (one-hop performance without spatial reuse), the performance gain by adaptively tuning the PCS threshold is significant $(200 \%$ in $8 \times 8$ grid and $250 \%$ in $10 \times 10$ grid).

We next evaluate the proposed adaptive PCS scheme, taking heterogeneous links into consideration. 100 nodes are randomly distributed in a $45 \times 45 \mathrm{~m}^{2}$ area as shown in Fig.10 a). Fig. $10 \mathrm{~b}$ ) illustrates the aggregate one-hop throughput achieved with different static PCS threshold values. Dashed horizontal lines represent the average aggregate throughput achieved by automatically configuring the carrier sense threshold with the Adaptive PCS scheme. The results clearly demonstrate that the adaptive PCS scheme achieves near-optimal performance even in the case of random topology with heterogeneous links.

All the work so far has only considered a deterministic path loss model to relate the received power as a function of T-R separation. Clearly, this is far from satisfactory and so as a final step, we take the impact of shadow fading into consideration and investigate it's impact on the adaptive PCS 
scheme performance. Here we revert back to a 10x10 regular grid as in Fig.7 to remove the effects of random topology.

Lognormal shadow fading on a link is modelled by modifying Eq.1 as follows:

$$
P_{r x}(d)=\bar{P}_{r x}-10 \gamma \log _{10}(d / \bar{d})+X \quad(\text { all powers in } \mathrm{dB}),
$$

where $X$ is a Gaussian random variable (also called fading factor) with zero mean and variance $\sigma^{2}$. Usually, the fading should be on a small scale (say millisecond), i.e. $\mathrm{X}$ is randomly chosen for every millisecond ( $\mathrm{ms}$ ). However, this will dramatically increase the complexity of simulation and slow down the speed. Here, we use the packet level energy calculation that allows simpler and faster simulation of a lognormal fading channel with $\sigma$ on the range $(0$, $5)$.

Fig.11 shows that the performance of the adaptive PCS degrades as the deviation $\sigma$ increases, which is reasonable since the prediction of interference level by energy sensing becomes less and less dependable with $\sigma$ increasing. For comparison, we also demonstrated the performance of the static PCS with the PCS threshold manually set as the optimal value $(-11 \mathrm{~dB})$, showing that near-optimal performance can be still achieved by the adaptive scheme even in a fading environment. One solution to the performance degradation is to lengthen the sensing duration DIFS, but the side effect of using longer DIFS is the increased overhead. Nevertheless, unless the DIFS exceeds the transmission time of RTS/CTS handshaking, the overhead of PCS will not be higher than VCS, hence PCS threshold adaptation is a viable option.

\subsection{Remark on Implementation Aspects}

As stated in IEEE 802.11 standards [1], the task of physical carrier sensing is carried by the Clear Channel Assessment (CCA). There are three CCA modes available for 802.11 DSSS (Direct Sequence Spread Spectrum) PHY:

"CCA Mode 1: Energy above threshold. CCA shall report a busy medium upon detection of any energy above the Energy Detection (ED) threshold.

CCA Mode 2: Carrier sense only. CCA shall report a busy medium only upon detection of a DSSS signal. This signal may be above or below the ED threshold.

CCA Mode 3: Carrier sense with energy above threshold. CCA shall report a busy upon detection of a DSSS signal with energy above the ED threshold." (page 223 in [1]) 
Clearly the ED threshold in the CCA Mode 1 has the same physical meaning as the PCS threshold in this paper, and can be used for enhanced physical carrier sensing. Unfortunately, the idea of enhancing PCS to mitigate interference via tuning the threshold does not appear to have attracted significant industry or academic attention, at least in relation to the literature on VCS based approaches. As a consequence, the ED threshold is not tunable or even accessible in most current 802.11 chips. However, a few vendors do allow ED threshold tuning, namely the Prism 2.5 ISL3873B ${ }^{7}$ chipset, for example. ISL3873B has a 8-bit register to configure the ED threshold, where Bit 7 is ED threshold control: $0=$ threshold is relative to noise floor and $1=$ threshold is absolute, and Bits $6 \sim 0$ is the ED threshold that could be set to any value on the range $[0,127](\mathrm{dBm})$.

\section{Conclusion}

In this paper, we proposed to enhance physical carrier sensing with a dynamically tunable sensing threshold to improve spatial reuse in 802.11 mesh networks, to increase the aggregate network throughput. Simulations were performed for both 1-D chain and 2-D grid topologies to validate the analysis and the proposed scheme. The main contributions of this paper are:

(1) We first provided a simple estimate of the optimal PCS threshold for maximizing the aggregate network throughput in a homogeneous mesh network and was validated by simulations.

(2) Next, we demonstrated an adaptive PCS scheme intended for heterogeneous networks where the PCS threshold is tuned by individual nodes based on local carrier sensing to achieve a substantial aggregate throughput improvement.

\section{References}

[1] IEEE Standard for Wireless LAN Medium Access Control (MAC) and Physical Layer (PHY) specifications, ISO/IEC 8802-11: 1999(E), Aug. 1999.

\footnotetext{
7 http://www.intersil.com
} 
[2] B. P. Crow, J. G. Kim, IEEE 802.11 Wireless Local Area Networks, IEEE Comm. Mag., Sept. 1999.

[3] G. Bianchi, Performance Analysis of the IEEE 802.11 Distributed Coordination Function, IEEE JSAC, vol. 18, no. 3, March 2000.

[4] X. Guo, S. Roy, W. Steven Conner, Spatial Reuse in Wireless Ad-Hoc Networks, VTC2003.

[5] J. Medbo and J.-E. Berg, Simple and accurate path loss modeling at 5 $\mathrm{GHz}$ in indoor environments with corridors, IEEE VTS-Fall VTC 2000. 52nd, Volume: 1, 24-28 Sept. 2000.

[6] X. Guo, "Personal Communication", 2003.

[7] Z. Li, S. Nandi, and A. K. Gupta, Improving Fairness in IEEE 802.11 based MANETs using Enhanced Carrier Sensing, http://www.ntu.edu.sg/home5/pg03802331/papers/ecs.pdf.

[8] D. Shukla, L. Chandran-Wadia, S. Iyer, Mitigating the exposed node problem in IEEE 802.11 adhoc networks, IEEE ICCCN 2003, Dallas, Oct 2003.

[9] F. A. Tobagi, L. Kleinrock, Packet Switching in Radio Channels: PART II- The Hidden Terminal Problem in Carrier Sensing Multiple Access and Busy Tone Solution", IEEE Trans. on Commun, Vol. COM-23, No. 12, pp. 1417-1433, 1975.

[10] K. Xu, M. Gerla, S. Bae, How effective is the IEEE 802.11 RTS/CTS handshake in ad hoc networks?, GLOBECOM02, Nov 17-21, 2002.

[11] S. Xu, T. Saadawi, Does the IEEE 802.11 MAC Protocol Work Well in Multihop Wireless Ad Hoc Networks? IEEE Communications Magazine, P130-137, June 2001.

[12] F. Ye, B. Sikdar, Improving Spatial Reuse of IEEE 802.11 Based Ad Hoc Networks, IEEE GLOBECOM, San Francisco, December 2003.

[13] http://www.opnet.com

[14] Intersil, Direct Sequence Spread Spectrum Baseband Processor, Doc\# FN 4816.2, Feb. 2002, http://www.intersil.com/ 
[15] V.Bharghavan, A. Demers, S. Shenker, and L. Zhang, MACAW: A Media Access Protocol for Wireless LANs, in Proc. Of ACM SIGCOMM'94,

[16] A.J. van der Vegt, Auto rate fallback algorithm for the ieee 802.11a standard, http://www.phys.uu.nl/ vdvegt/docs/gron/.

[17] Theodore S. Rappaport. Wireless Communications, Principles and Practices, 2nd Ed. Prentice Hall, 2002. ISBN 0-13-042232-0

[18] "PRISM 2.5 Wireless LAN Integrated Medium Access Controller with Baseband Processor", http://www.intersil.com/data/fn/fn8019.pdf 


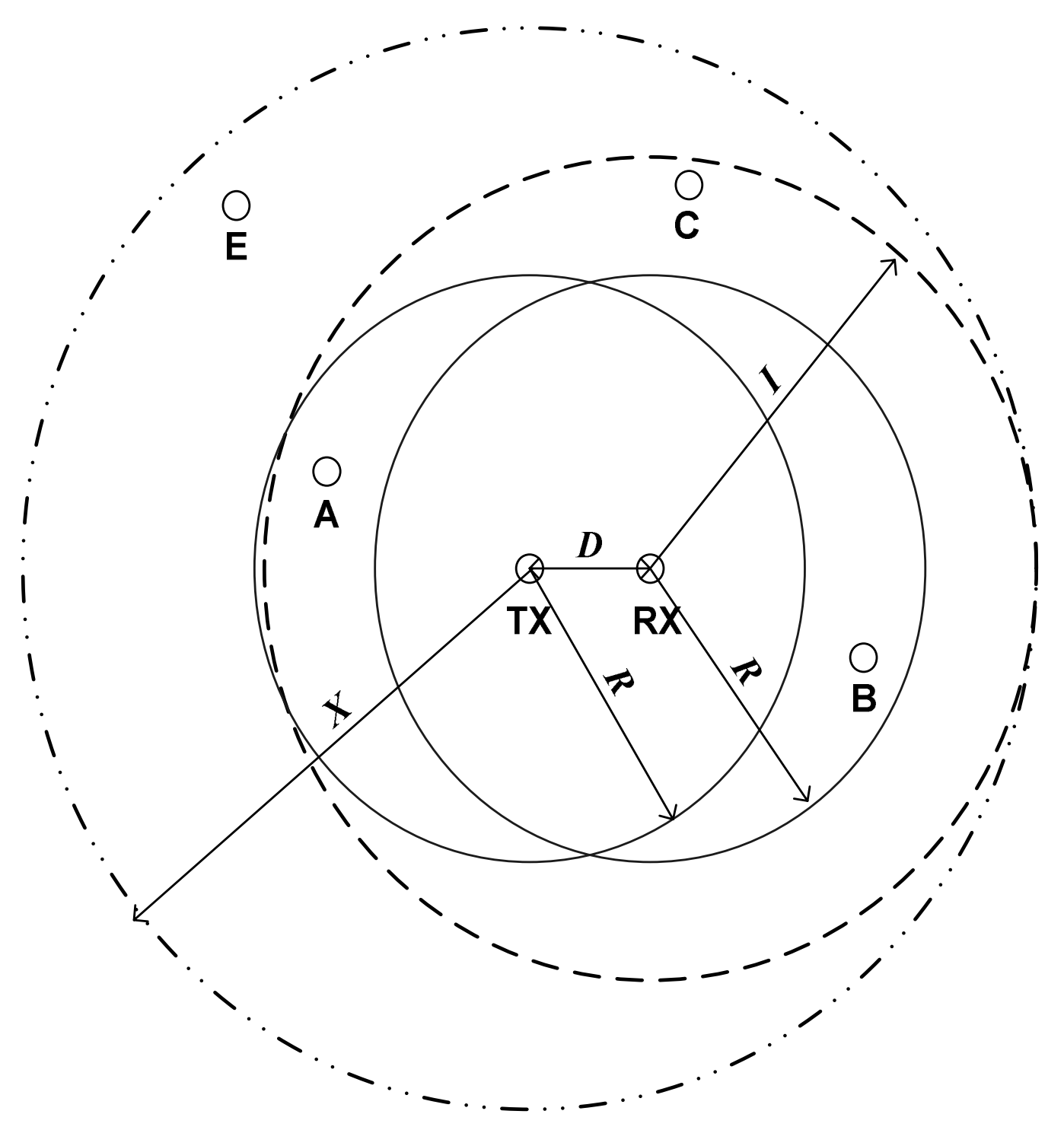

Fig. 1: Illustration of relative transmission and interference distances in a wireless mesh network 


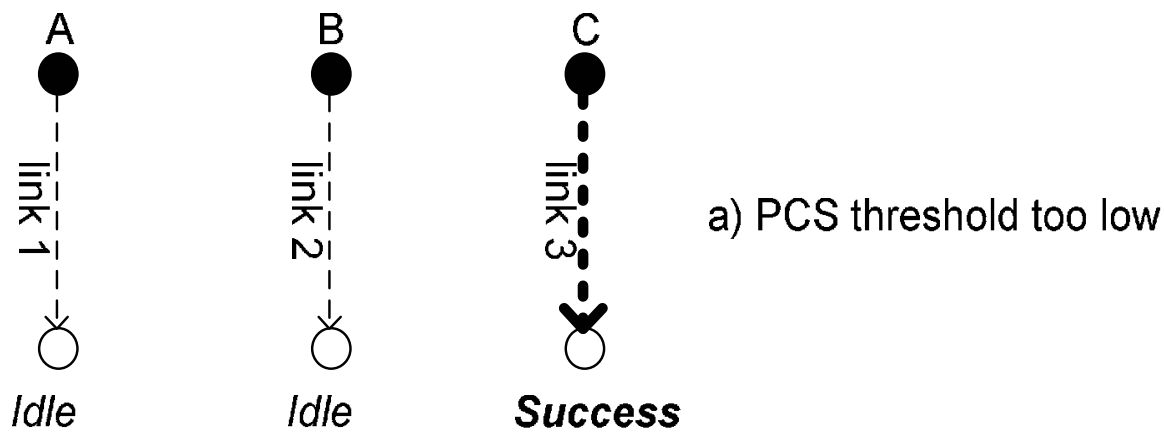

High interference disrupts communication.

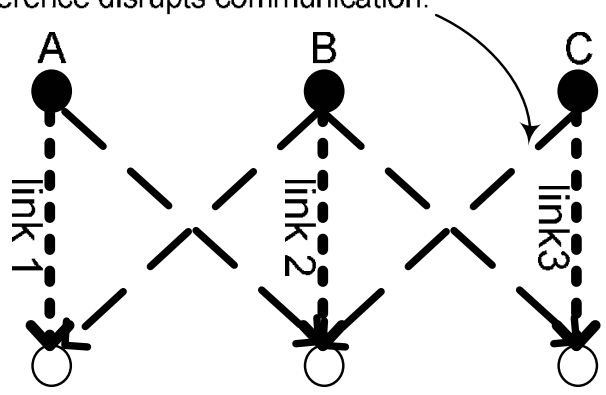

b) PCS threshold too high

Collision Collision Collision

Low interference overcome by high SNR



Fig. 2: Physical Carrier Sensing (PCS) and Spatial Reuse. 




Fig. 3: 802.11 MAC ACK Head 


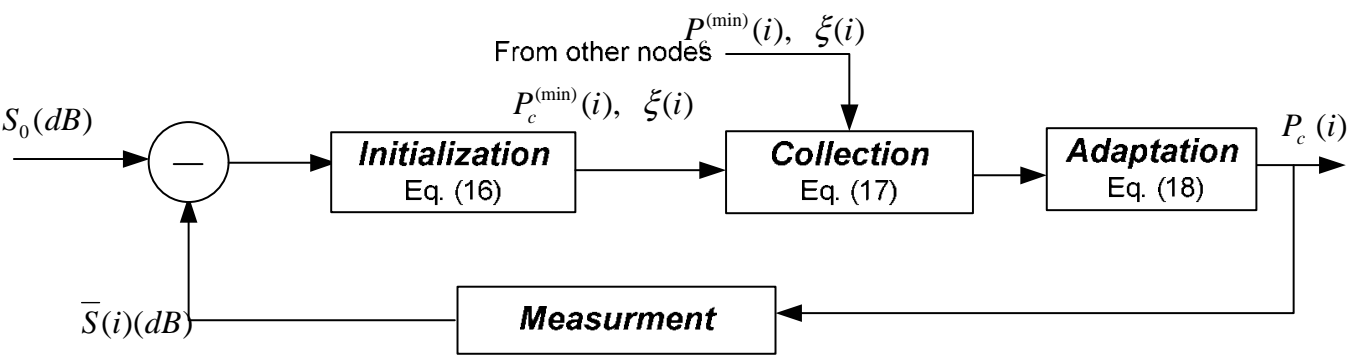

Fig. 4: Feedback Control Diagram of the Estimation-based Adaptive Physical Carrier Sensing 


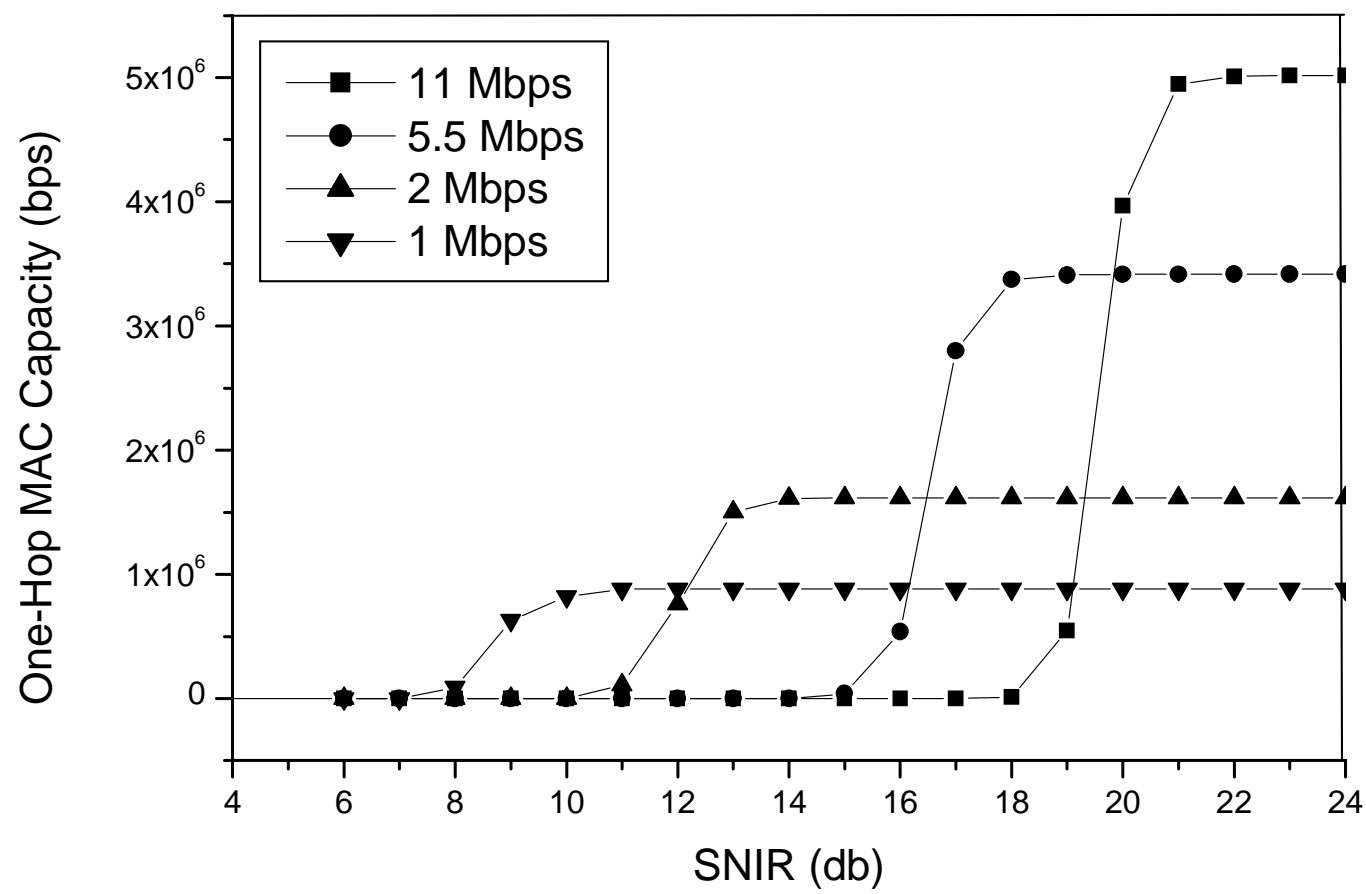

Fig. 5: One-Hop multi-rate performance of $802.11 \mathrm{~b}$ for various SNIR values at the receiver (RTS/CTS disabled). 


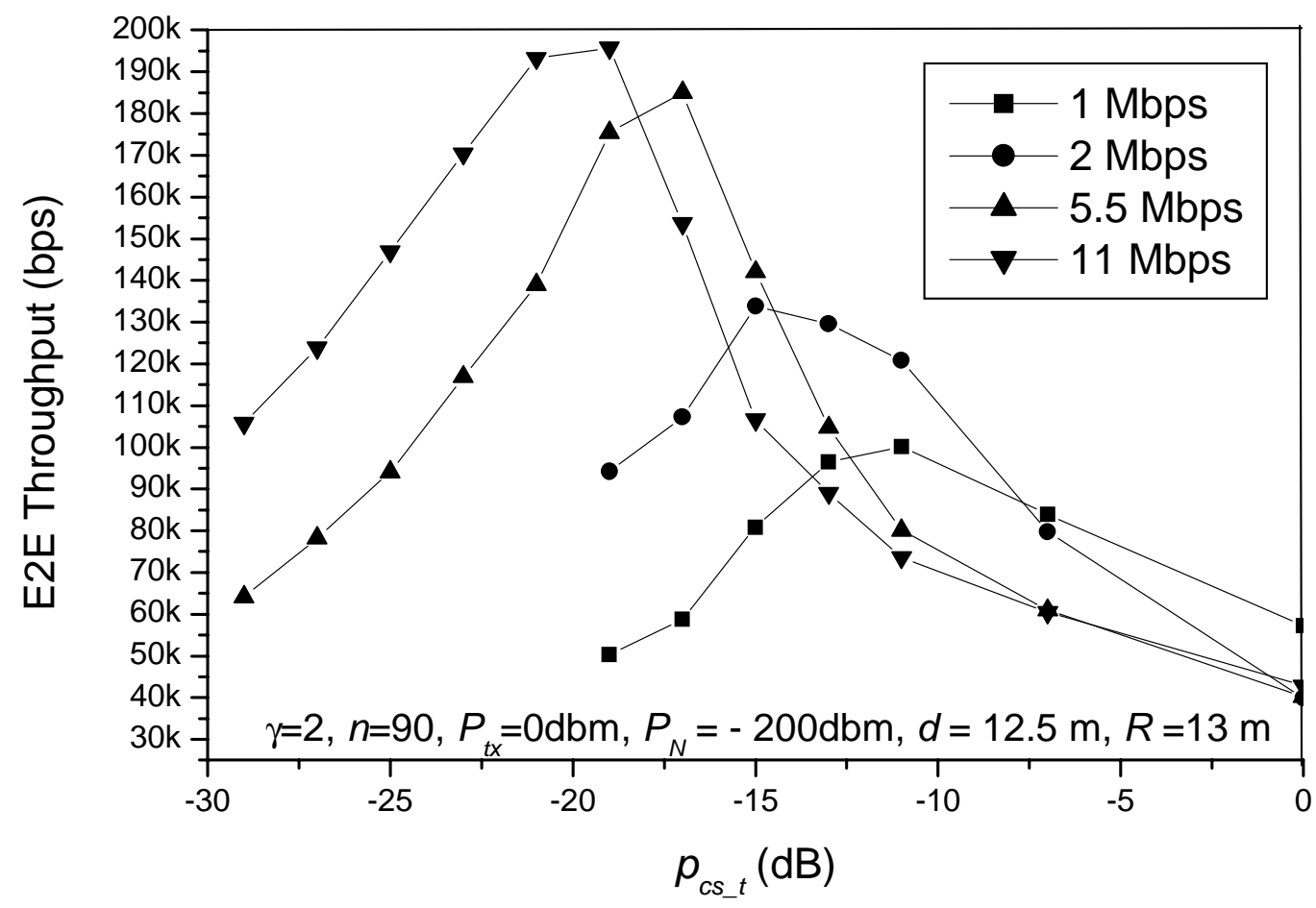

Fig. 6: End-to-end throughput in a 90-hop chain for various sensing thresholds and data rates. 


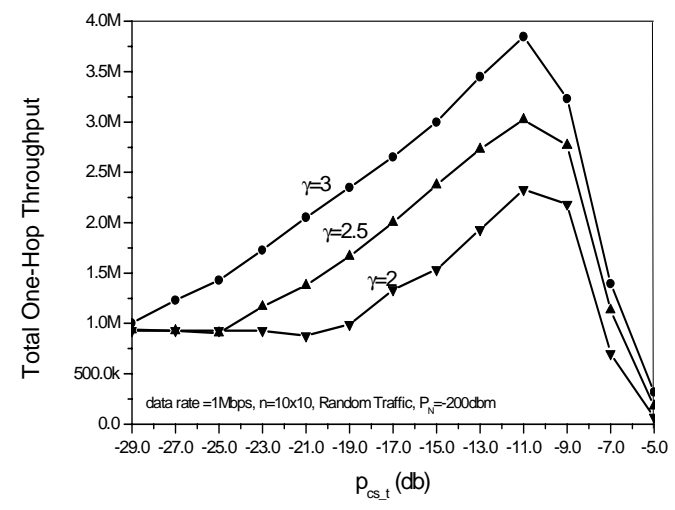

a) Data Rate $=1 \mathrm{Mbps}$

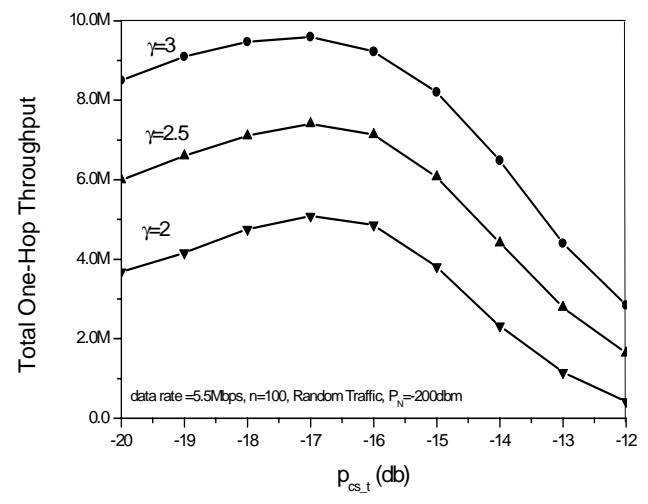

c) Data Rate $=5.5 \mathrm{Mbps}$

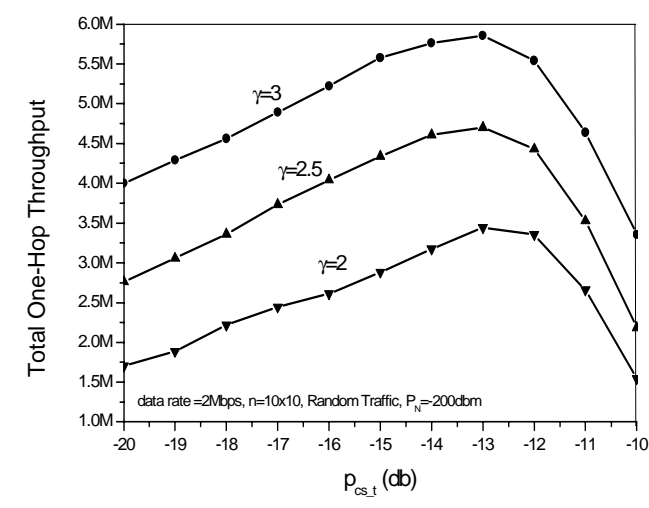

b) Data Rate $=2 \mathrm{Mbps}$

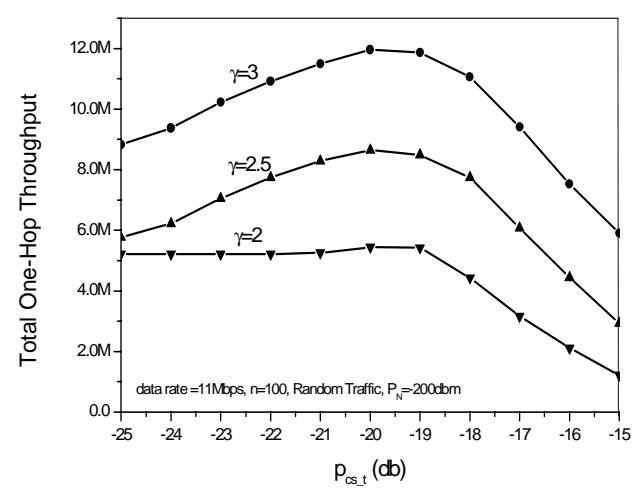

d) Data Rate $=11 \mathrm{Mbps}$

Fig. 7: Aggregate 1-hop throughput as a function of PCS threshold for various pathloss exponent values in a $10 \times 10802.11$ b grid 


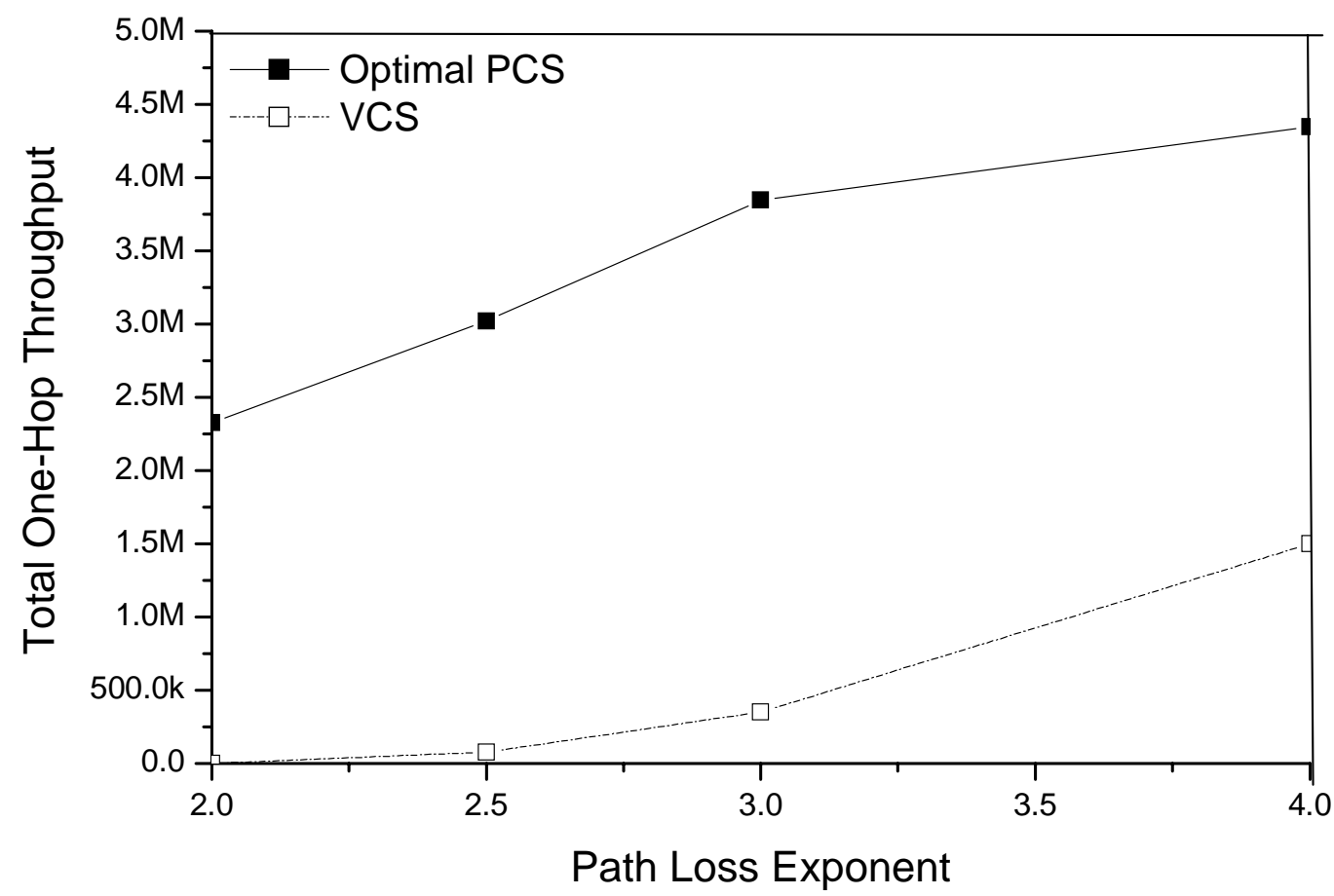

Fig. 8: Optimal Physical Carrier Sensing vs. Virtual Carrier Sensing with RTS $/$ CTS (data rate $=1 \mathrm{Mbps}$ ) 


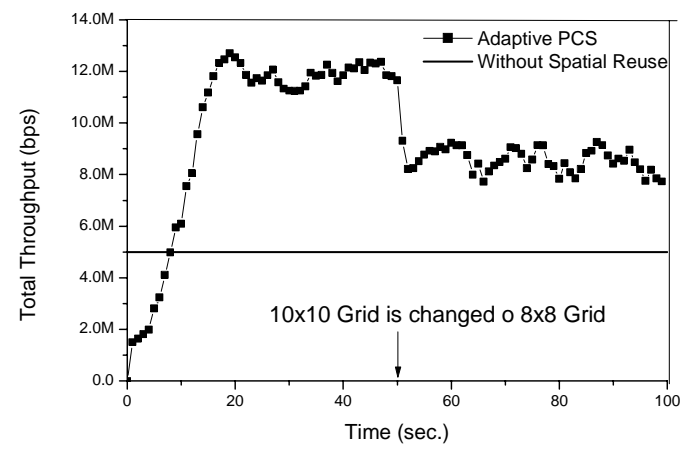

a) Tracing Throughput

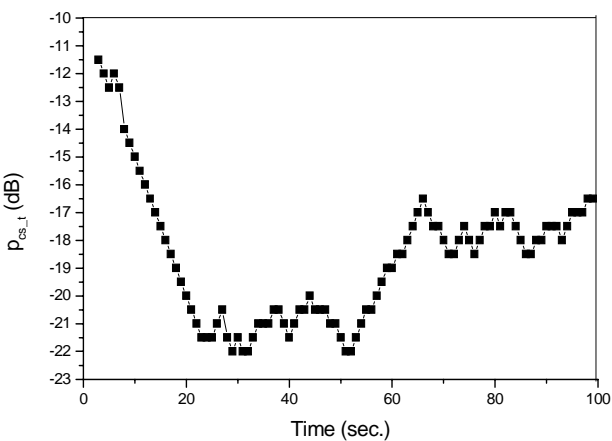

b) Tracing PCS threshold

Fig. 9: Effect of automatic APCS adaptation when network topology changes $($ Data Rate $=11 \mathrm{Mbps}, \gamma=3$ ) 



Fig. 10: Performance of the Adaptive PCS in a Random Topology: a) Topology and b) Simulation Results 


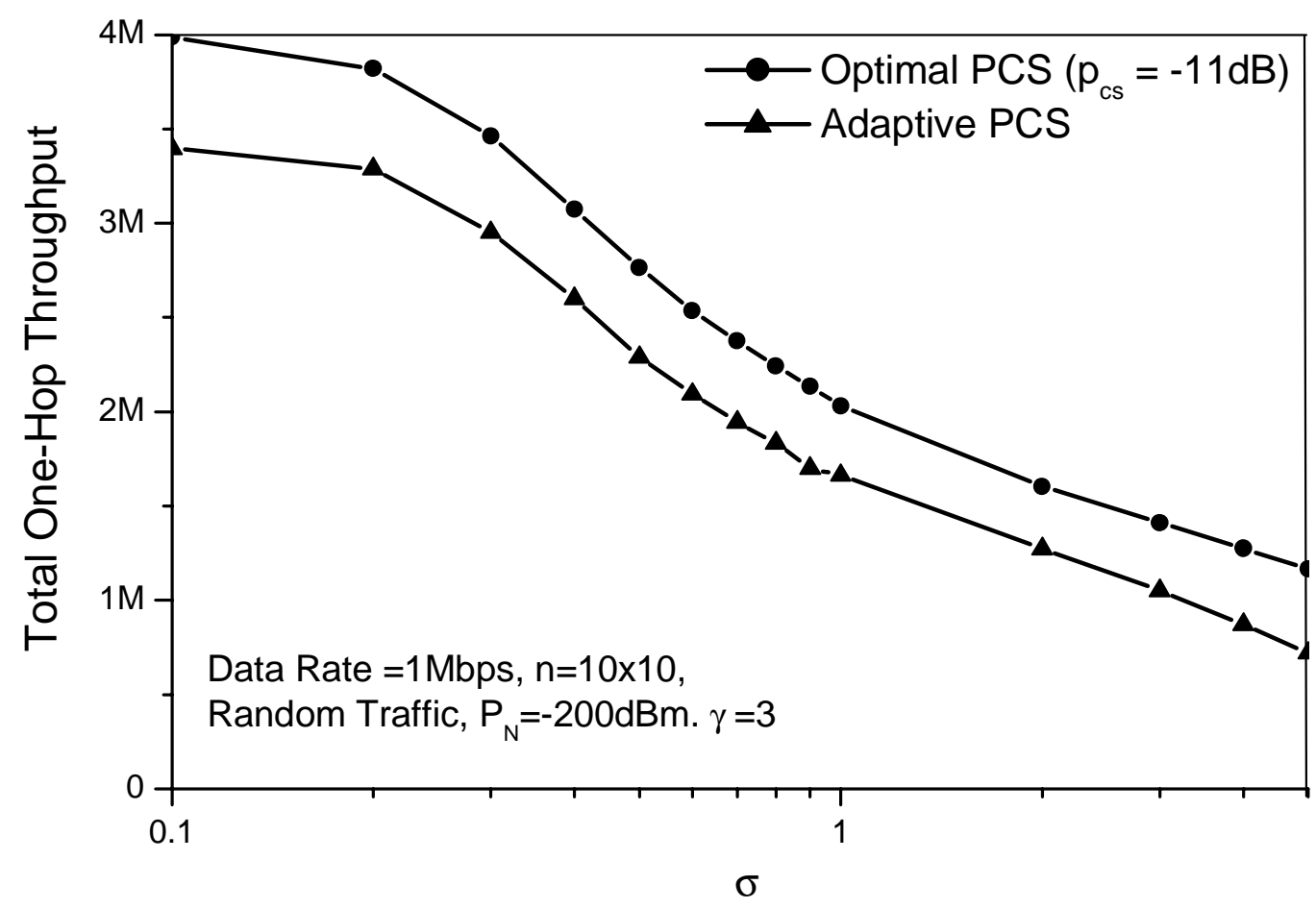

Fig. 11: Effect of Dynamic Channel Variation Due to Fading on the Performance of Adaptive PCS 